\title{
ZMP Trajectory Generation for Reduced Trunk Motions of Biped Robots
}

\author{
Jong H. Park \\ School of Mechanical Engineering \\ Hanyang University \\ Seoul, 133-791, Korea \\ email:jong.park@ieee.org
}

\author{
Yong K. Rhee \\ School of Mechanical Engineering \\ Hanyang University \\ Seoul, 133-791, Korea
}

\begin{abstract}
Trunk motions are typically used to stabilize the locomotion of biped robots, which can be very large in some leg trajectories. This paper proposes a method to reduce the motion range of the trunk by generating a desired trajectory of the ZMP. The trajectory is determined by a fuzzy logic based upon the leg trajectories that are arbitrary selected. The resulting ZMP trajectory is similar to human's one and the ZMP continuously moves forward. The proposed scheme is simulated on a 7-degree-of-freedom biped robot. Its results indicate that the proposed ZMP trajectory increases the stability of the locomotion and thus resulting in reduction of motion range of the trunk.
\end{abstract}

\section{Introduction}

Generating desired trajectories for dynamic walking of biped robots is one of the important research areas. The simplest method to generate trajectories is the inverted pendulum mode $[1,2]$. However, when the trajectory is implemented, the stability of the biped robot is compromised by the masses that are assumed to be negligible in generating the trajectory. There exists a method to keep the simplicity of the inverted pendulum mode while improving the stability [3]. However, all these methods are not flexible in generating arbitrary leg motions of the biped robot.

A more complicated but more accurate method is based upon the zero-moment point (ZMP) equation, which describes relationship between the joint motions and the forces applied at the ground $[4,5,6]$. Yamaguchi et al. [5] and Li et al. [7] used trunk swing motions and trunk yaw motions, respectively, to increase the locomotion stability for an arbitrary robot loco- motion. It is very important to coordinate the upper body and the lower legs in order to stabilize dynamic locomotions of the biped robot. Another work by [8] controls the actual ZMP to track a reference ZMP.

However, previous researches have assumed that the ZMP does not change at all or that ZMP changes in an uncoordinated fashion during walking as long as one foot is on the ground. The human has more stable and adaptive locomotion by changing his ZMP appropriately. We propose in this paper that the ZMP should be changed as the body moves during a stride. It is based upon observations of human locomotion, where the heel of the free leg contacts the ground first, and then its middle part and finally its toe contact the ground. In the proposed scheme, the contact point with the ground, i.e., the ZMP, moves continuously forward as the locomotion progresses. The desired ZMP trajectory depends upon the robot configuration, especially hip position and the swinging free leg.

We implemented a fuzzy-logic algorithm to generate the desired ZMP trajectory depending upon the body posture. The control scheme is simulated only in the sagittal plane. In this paper, locomotion only with the single-leg-support phase, where only one leg supports the weight of the biped robot, is considered.

Section 2 explains the ZMP equation and Section 3 describes the desired ZMP trajectory and the fuzzy logic to generate it along with overall control system configuration. Section 4 covers computer simulations of the proposed control scheme and their results, followed by conclusions in Section 5 .

\section{ZMP Equation}

Suppose that the ground applies a pure force to the biped robot at a location $p$, called the ZMP, which 


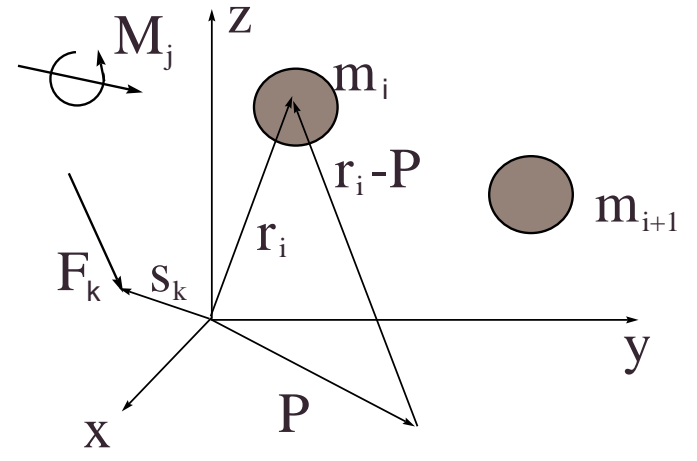

Figure 1: The coordinate frame and the ZMP.

should be within the foot of the supporting leg for the biped robot to be stable. Then the dynamics of the biped robot under other external forces and moments becomes

$$
\begin{aligned}
\sum_{i} m_{i}\left(r_{i}-p\right) \times\left(-\ddot{r}_{i}\right. & +g)+\sum_{j} M_{j} \\
& +\sum_{k}\left(s_{k}-p\right) \times F_{k}=0
\end{aligned}
$$

where $m_{i}$ is the mass of the link $i ; r_{i}$ is the position of the mass center of link $i ; M_{j}$ is an external moments applied to the biped robot; $s_{k}$ is the location where external force $F_{k}$ is applied; and

$$
\begin{aligned}
g & =\left[\begin{array}{lll}
g_{x} & g_{y} & g_{z}
\end{array}\right]^{T} \\
r_{i} & =\left[\begin{array}{lll}
x_{i} & y_{i} & z_{i}
\end{array}\right]^{T} \\
p & =\left[\begin{array}{lll}
x_{z m p} & y_{z m p} & 0
\end{array}\right]^{T} \\
F_{k} & =\left[\begin{array}{lll}
F_{x, k} & F_{y, k} & F_{z, k}
\end{array}\right]^{T} \\
s_{k} & =\left[\begin{array}{lll}
x_{k} & y_{k} & z_{k}
\end{array}\right]^{T} \\
r_{i} & =\left[\begin{array}{lll}
x_{i} & y_{i} & z_{i}
\end{array}\right]^{T} \\
M_{j} & =\left[\begin{array}{lll}
M_{x, j} & M_{y, j} & M_{z, j}
\end{array}\right]^{T} .
\end{aligned}
$$

Note that in normal locomotion on a horizontal surface, $g_{z}=-g$. See Fig. 1 .

Considering the dynamics in the $y$-direction only under the assumption of no external moments, i.e., $M_{j}=0$, Eq. (1) can be expressed as

$$
\begin{aligned}
x_{z m p}= & \frac{\sum_{i=1}^{n} m_{i}\left(\ddot{z}_{i}-g_{z}\right) x_{i}-\sum_{i=1}^{n} m_{i}\left(\ddot{x}_{i}-g_{x}\right) z_{i}}{\sum_{i=1}^{n} m_{i}\left(\ddot{z}_{i}-g_{z}\right)-\sum_{k} F_{z, k}} \\
& +\frac{\sum_{k}\left(z_{k} F_{x, k}-x_{k} F_{z, k}\right)}{\sum_{i=1}^{n} m_{i}\left(\ddot{z}_{i}-g_{z}\right)-\sum_{k} F_{z, k}} .
\end{aligned}
$$

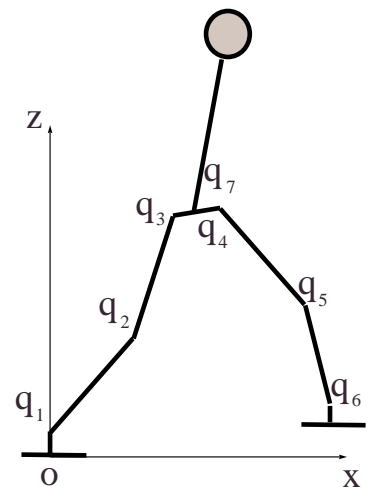

Figure 2: Model of a 7 degree-of-freedom biped robot.

where $n$ is the total number of components of the robot.

\section{$3 \quad$ ZMP Trajectory Control}

\subsection{ZMP Trajectory}

Motions of the biped robot can be considered as two parts: leg motions and the trunk (or upper-body) motions. Leg motions should be determined depending upon its environment. For example, if there is an obstacle is in the way of the swinging free leg, the foot of the free leg should be lifted up higher. Or if the obstacle is at the location where the free leg is to land on the ground, the landing point and thus the walk pace should be modified. In general, terrain environment is not known a priori, it should be possible to change the walking pattern anytime.

On the other hand, it is desired that the posture of the trunk is kept stationary. This allows, for example, the robot to carry objects in a stable manner, or to get scenery information with vision cameras. In normal locomotion, the trunk should be in a straight-up position. When the biped robot climbs up a hill, its trunk should lean forward to increase the stability of locomotion.

Assuming that the trajectory of the legs are already determined, for example, by the terrain constraints, we are to determine the trunk motion that would keep its stationary posture. Figure 2 shows the model of the biped robot to be considered here.

Assuming that the height of the trunk is constant because of the stationary posture, i.e., $\ddot{z}_{7}=\dot{z}_{7}=0$, 

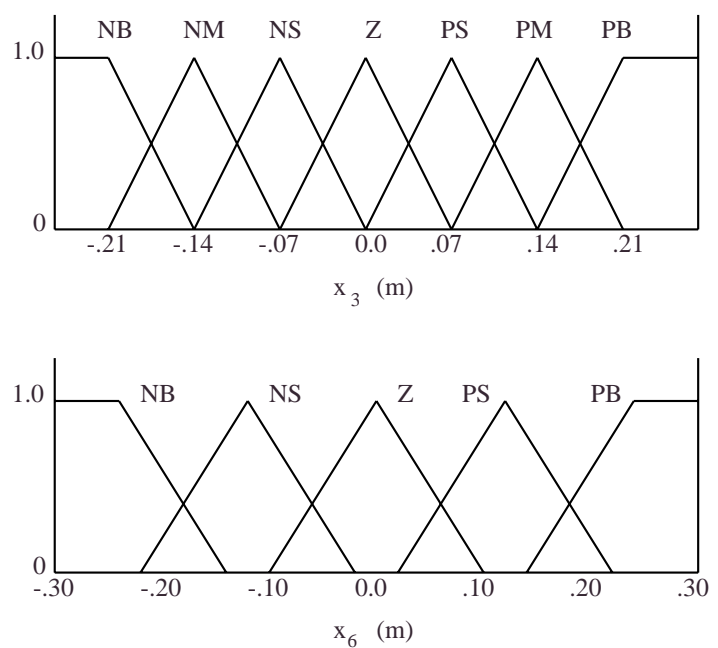

Figure 3: Fuzzy membership functions for $x_{3}$ and $x_{6}$.

Eq. (2) becomes

$$
\begin{aligned}
m_{7} \ddot{x}_{7} z_{7}+m_{7} g_{z} x_{7}=\sum_{i=1}^{6} m_{i}\left(\ddot{z}_{i}-g_{z}\right) x_{i} \\
-\sum_{i=1}^{6} m_{i}\left(\ddot{x}_{i}-g_{x}\right) z_{i}+m_{7} g_{x} z_{7} \\
+x_{z m p}\left\{m_{7} g_{z}-\sum_{i=1}^{6} m_{i}\left(\ddot{z}_{i}-g_{z}\right)+\sum_{k} F_{z, k}\right\} .
\end{aligned}
$$

If the ZMP is determined and no external force exists, the right-hand side terms of the equation are known except for external forces and thus differential equation of Eq. (3) can be easily solved numerically.

\subsection{Fuzzy Algorithm for the ZMP}

When a human walks, the ZMP is not fixed but rather moves. In the human locomotion, it is at the heel that the foot of the free leg contacts the ground first. And then the middle part of the foot and finally its toe come into a contact with the ground. This indicates that a human uses strategy of moving the ZMP continuously forward as the locomotion progresses. Based upon this observation, we implemented a fuzzy logic to generate the desired ZMP trajectory $x_{z m p}(t)$ which depends upon the configuration of the biped robot. The rule base of the logic is shown in Table 1.

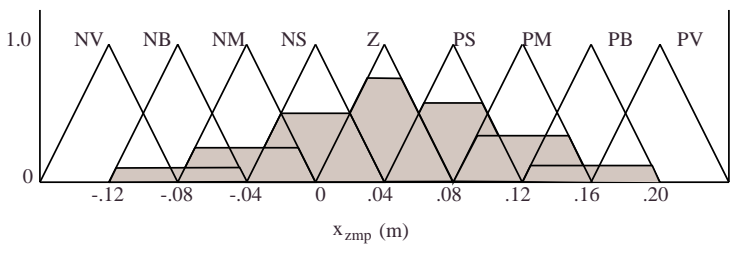

Figure 4: Fuzzy membership functions for $x_{z m p}$ and the defuzzification using the center of gravity.

Table 1: The rule base to generate the desired ZMP trajectory depending upon the hip and the free leg positions.

\begin{tabular}{|c||c|c|c|c|c|c|c|}
\hline \multicolumn{1}{|c||}{$x_{6}$} & \multicolumn{7}{c|}{$x_{3}$} \\
\cline { 2 - 8 } & PB & PM & PS & Z & NS & NM & NB \\
\hline \hline PB & PV & PV & PB & PS & NS & NM & NB \\
\hline PS & PV & PB & PM & Z & NS & NB & NB \\
\hline Z & PV & PB & PM & Z & NM & NB & NV \\
\hline NS & PB & PB & PS & Z & NM & NB & NV \\
\hline NB & PB & PM & PS & NS & NB & NV & NV \\
\hline
\end{tabular}

The desired ZMP is greatly influenced by the position of the hip, $x_{3}$, and in a less degree by the position of the free leg configuration, which is represented by the foot position of the free leg, $x_{6}$. Five fuzzy membership functions are used for each of $x_{3}$ and $x_{6}$ as shown in Fig. 3. The logic shows that the more the hip is in front of the supporting foot, the more positive the ZMP becomes. A similar relationship exists between the free leg and the ZMP in a less degree.

Total seven fuzzy membership functions are used for the ZMP as shown in Fig. 4. The asymmetry reflects a human foot, which the heel is slightly behind the ankle and the toe is farther away in front of the ankle. Defuzzification based upon the center of gravity is used.

\subsection{Joint Controller}

Based upon the ZMP computed by the fuzzy logic, and under the assumption that no external force is applied to the robot, we used the 4th-order Runge-Kutta method to solve Eq. (3) for $x_{7}$ with the desired ZMP trajectory generated by the fuzzy logic. Trunk trajectory $x_{7}(t)$ and leg trajectories $x_{i}(t)$, for $i=1, \ldots, 6$, determines the complete desired motion of the biped robot. The desired joint trajectory $q_{d}(t)$ is then com- 
puted using the inverse kinematics of the robot.

We used the computed-torque control to track the desired joint trajectory. The dynamics of the biped robot is expressed as

$$
M(q) \ddot{q}+N(q, \dot{q})=\tau+J^{T} F
$$

where $F$ denotes an external force, and $N(q, \dot{q})$ denotes Coriolis and centrifugal terms. Joint torque $\tau$ is then computed as

$$
\tau=M(q)\left(\ddot{q}_{d}+k_{v} \dot{e}+k_{p} e\right)+N(q, \dot{q})
$$

where joint error $e(t)$ is defined as $e(t)=q_{d}(t)-q(t)$.

The overall control scheme for the biped robot is shown in Fig. 5. Note that external disturbance forces are not considered in this paper at all.

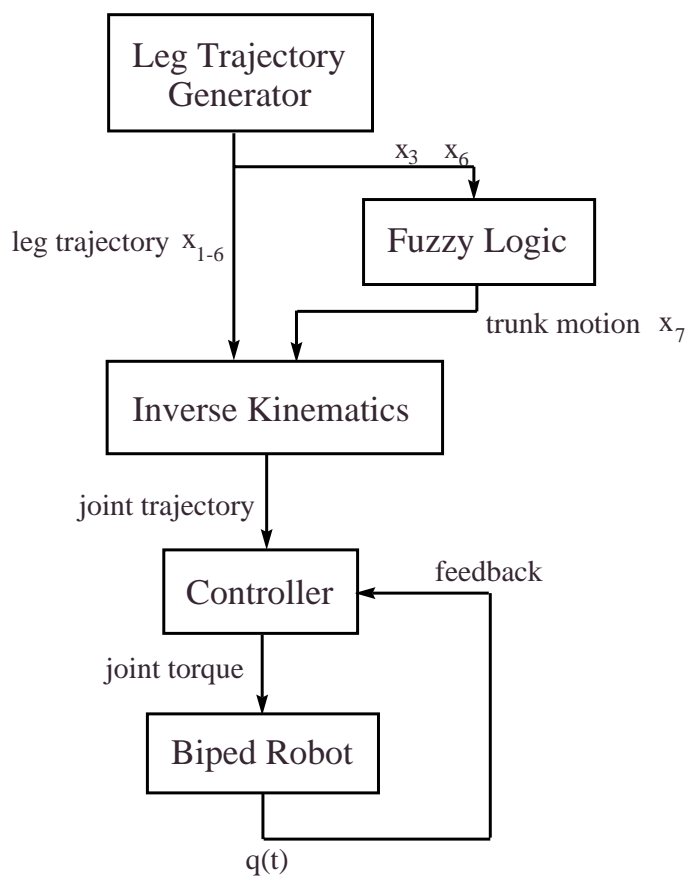

Figure 5: Overall control system block diagram.

\section{Simulations}

In a series of simulations, the control scheme proposed in this paper was compared with the conventional scheme based upon a fixed ZMP. Simulations were done for different locomotion patterns, i.e., different leg motions, Range of the trunk motions required
Table 2: Masses and dimensions of the biped robot links.

\begin{tabular}{|c|c|c|c|}
\hline Link & $\begin{array}{c}\text { Length } \\
(\mathrm{m})\end{array}$ & $\begin{array}{c}\text { Mass } \\
(\mathrm{kg})\end{array}$ & $\begin{array}{c}\text { Inertia Moment } \\
\left(\mathrm{kgm}^{2}\right)\end{array}$ \\
\hline Link 1 & 0.05 & 3 & 0.01 \\
\hline Link 2 & 0.40 & 5 & 0.05 \\
\hline Link 3 & 0.40 & 5 & 0.05 \\
\hline Link 4 & 0.40 & 5 & 0.05 \\
\hline Link 5 & 0.40 & 5 & 0.05 \\
\hline Link 6 & 0.05 & 3 & 0.01 \\
\hline Link 7 & 0.60 & 57 & 0.13 \\
\hline
\end{tabular}

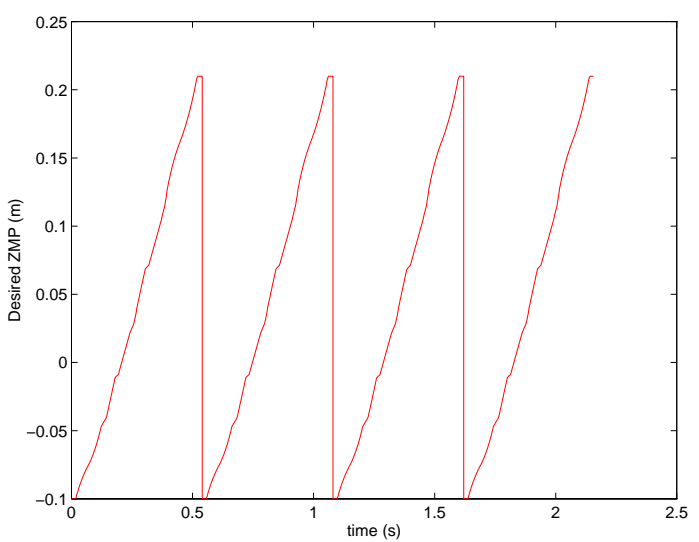

Figure 6: The desired ZMP trajectory moves forward.

to stabilize the biped robot is used as the stability measure. It is certainly not desirable to have a control scheme where the trunk has to swing back and forth in a large range in order to stabilize the biped locomotion. Thus, it can be assumed that the smaller the range of the swing motion, the more stable the control strategy is.

The biped robot used in the simulations is 1.45 $\mathrm{m}$ tall and weights $83 \mathrm{~kg}$. Detailed dimensions and masses of its links are shown in Table 2.

In the first simulation, the biped robot walks on a horizontal plane. The walking step is $0.30 \mathrm{~m} / \mathrm{step}$ and its speed is about $0.56 \mathrm{~m} / \mathrm{s}$. Based upon trajectories of the robot legs a priori, the desired ZMP trajectory is generated by the fuzzy logic, as shown in Fig. 6, where the ZMP moves forward relatively to the center of the supporting foot from $-0.07 \mathrm{~m}$ to $+0.15 \mathrm{~m}$. Figure 7 shows that the trunk motion range becomes far less when the ZMP trajectory generated by the fuzzy logic is implemented than when the fix ZMP is 


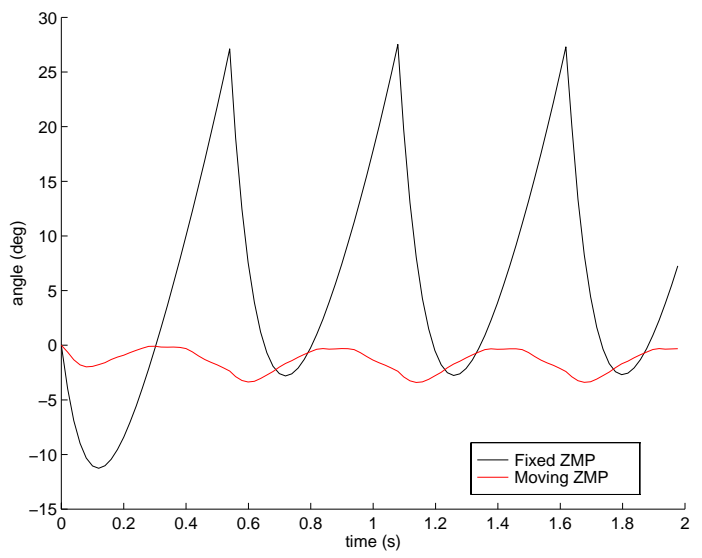

Figure 7: Trunk motions when the fixed and moving ZMP trajectories are used.

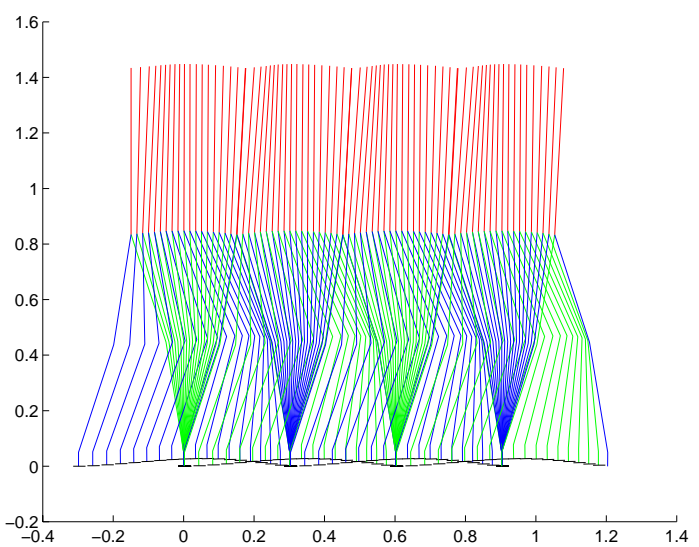

Figure 8: Walking simulation 1 with the moving ZMP.

implemented. Figure 8 and 9 show the walking simulation with the moving ZMP and with the fixed ZMP at $x=$, respectively. With the fixed ZMP, the trunk has to swing back and forth from $-10^{\circ}$ to $30^{\circ}$ while it moves less than $\pm 5^{\circ}$ with the moving ZMP. Figure 10 indicates the actual ZMP follows closely with the desired ZMP by the computed-torque controller.

Another simulation was done with the leg trajectories that are significantly different from the one used in the previous simulation. The simulation results are shown in Fig. 11 for the moving ZMP and Fig. 12 for the fixed ZMP. Even though the trunk motions generally become larger than the ones in the previous simulation, they are again smaller with the moving ZMP than with the fixed ZMP.

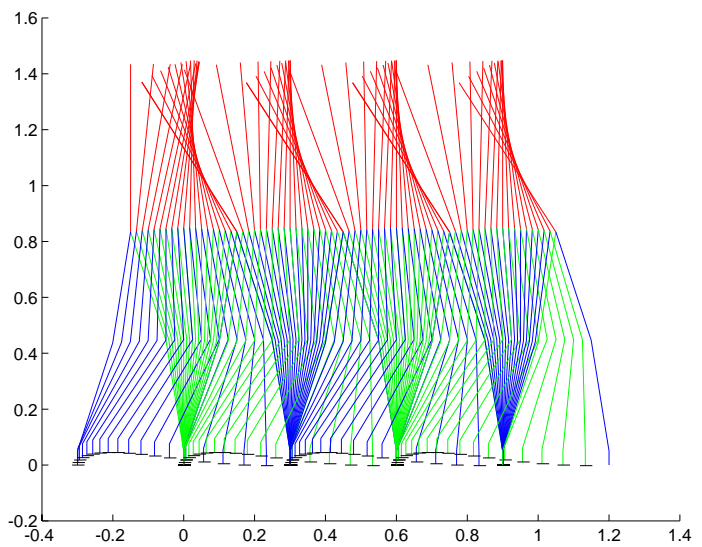

Figure 9: Walking simulation 1 with the fixed ZMP.

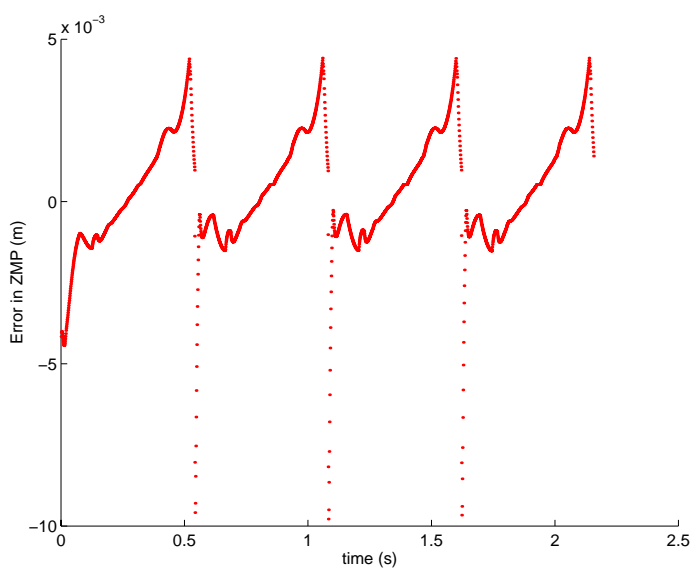

Figure 10: Errors in the ZMP trajectory.

\section{Conclusions}

This paper proposed a new control scheme for biped robots based upon a varying ZMP rather than a fixed ZMP. This scheme is very similar to what can be observed in the human locomotion. The ZMP trajectory produced by a fuzzy-logic algorithm moves continuously forward depending upon the hip position and the free leg configuration. The proposed scheme is simulated on a 7 degree-of-freedom biped robot and the simulation results show that it does not require a large swing motion of the trunk and thus significantly stabilizes the biped robot. 


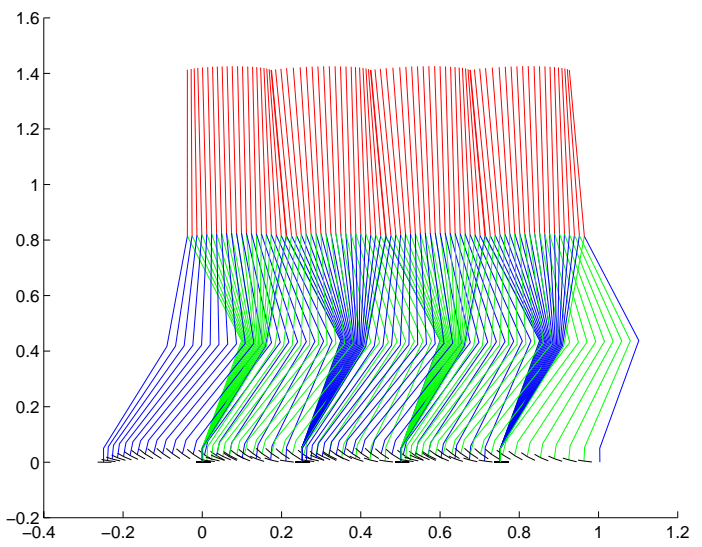

Figure 11: Walking simulation 2 with the moving ZMP.

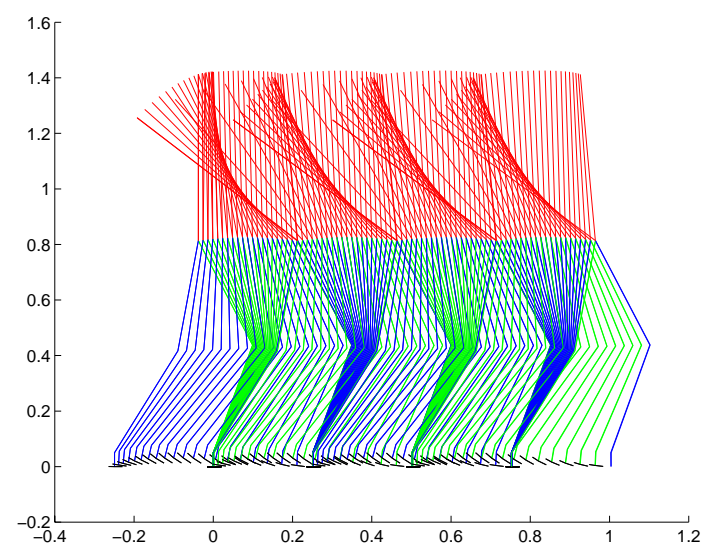

Figure 12: Walking simulation 2 with the fixed ZMP.

\section{Acknowledgments}

The authors would like to thank Prof. Takanish for his discussions with us.

\section{References}

[1] S. Kajita and K. Tani, "Study of dynamic biped locomotion on rugged terrain: Derivation and application of the linear inverted pendulum mode," in Proceedings of the IEEE International Conference on Robotics and Automation, (Sacramento, CA), pp. 1405-1411, 1991.

[2] S. Kajita and K. Tani, "Experimental study of biped dynamic walking in the linear inverted pen- dulum mode," in Proceedings of the IEEE International Conference on Robotics and Automation, (Nagoya, Japan), pp. 2885-2891, 1995.

[3] J. H. Park and K. D. Kim, "Biped robot walking using gravity-compensated inverted pendulum mode and computed torque control," in Proceedings of the IEEE International Conference on Robotics and Automation, (Lueven, Belgium), 1998.

[4] A. Takanish, M. Tochizawa, H. Karaki, and I. Kato, "Dynamic biped walking stabilized with optimal trunk and waist motion," in Proceedings of the IEEE/RSJ International Workshop on Intelligent Robotics and Systems, (Tsukuba, Japan), pp. 187-192, 1989.

[5] J. Yamaguchi, A. Takanish, and I. Kato, "Development of a biped walking robot compensating for three-axis moment by trunk motion," in Proceedings of the IEEE/RSJ International Workshop on Intelligent Robotics and Systems, (Yokohama, Japan), pp. 561-566, 1993.

[6] J. Yamaguchi, N. Kinoshita, A. Takanish, and I. Kato, "Development of a dynamic biped walking system for humanoid development of a biped walking robot adapting to the humans' living floor," in Proceedings of the IEEE International Conference on Robotics and Automation, (Minneapolis, MN), pp. 232-239, 1996.

[7] Q. Li, A. Takanish, and I. Kato, "Learning control of compensative trunk motion for biped walking robot based on ZMP stability criterion," in Proceedings of the IEEE/RSJ International Workshop on Intelligent Robotics and Systems, (Raleigh, NC), pp. 597-603, 1992.

[8] K. Sorao, T. Murakami, and K. Ohnishi, "A unified approach to ZMP and gravity center control in biped dynamic stable walking," in Proceedings of Advanced Intelligent Mechatronics, (Tokyo, Japan), 1997. 